This item was submitted to Loughborough's Institutional Repository (https://dspace.lboro.ac.uk/) by the author and is made available under the following Creative Commons Licence conditions.

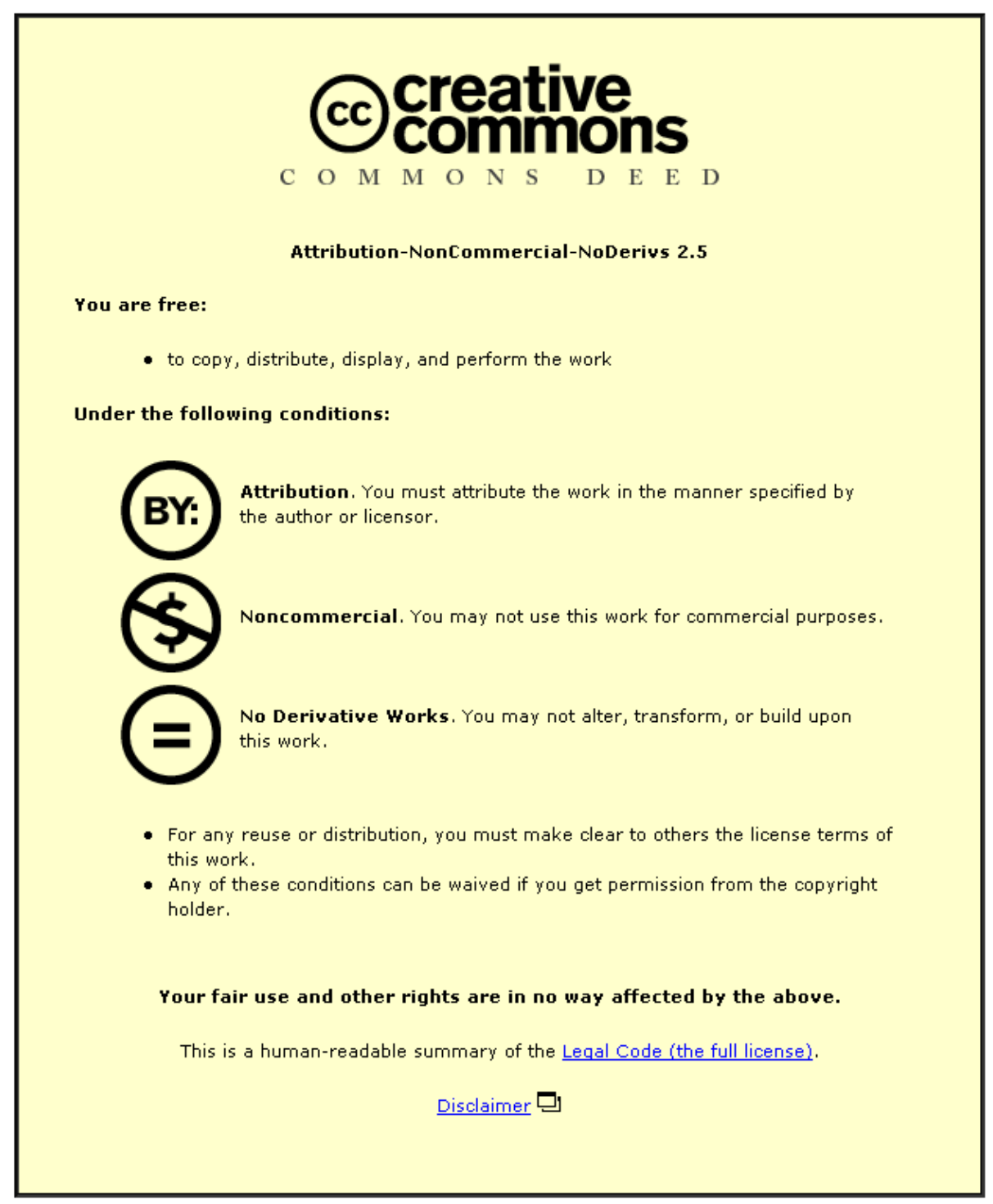

For the full text of this licence, please go to: http://creativecommons.org/licenses/by-nc-nd/2.5/ 


\title{
Strategic Planning for Water Utilities in Developing Countries
}

\author{
Josses Mugabi ${ }^{\mathrm{a}}$, Sam Kayaga ${ }^{\mathrm{a}^{\star}}$ and Cyrus $\mathrm{Njiru}^{\mathrm{a}^{\dagger}}$ \\ ${ }^{a}$ Water, Engineering and Development Centre, Loughborough University, Leicestershire LE11 3TU, UK
}

\begin{abstract}
A common feature of public water utilities in developing countries is their lack of a commercial orientation. As a result, many utilities find themselves locked in a cycle of poor corporate performance - with low coverage of services, huge amounts of nonrevenue water and insufficient funding for maintenance and expansion. Strategic planning in such turbulent times should be relevant, cost-effective and transforming. This paper discusses a strategic planning framework to assist utilities in developing meaningful and useful performance improvement plans. Recent application of this framework in Africa has demonstrated its relevancy, cost-effectiveness and potential to transform poorly performing water utilities.
\end{abstract}

Key words: Strategic planning; Urban Water Utilities; Developing Countries

\footnotetext{
* Corresponding Author: Tel: +44-1509-228743, Fax: +44-1509-211079:

Email: s.m.kayaga@lboro.ac.uk

${ }^{\dagger}$ Present Address: African Development Bank, BP 323-1002, Tunis Belvedere, Tunisia
} 


\section{Introduction}

Public water utilities in developing countries face enormous challenges in meeting the water needs of their growing urban populations. Many of these challenges are as a result of inappropriate utility management practices, including the lack of a commercial-oriented culture. A number of options have been tried in an attempt to address this problem. The most notable one is private sector participation - which has included contracting multinational water companies to run water utilities (World Bank, 2003). Although some developed countries may consider privatisation as the most viable option (Lam and Chan, 1998), private sector participation in developing countries has had only limited success, and there is growing pessimism about the scale of performance improvements to be expected from private sector involvement (Budds and McGranahan, 2003).

On the other hand, there is growing optimism that public water utilities in developing countries can improve their own performance by applying commercial management principles (Zuleta et al., 2005). One such principle that utilities can adapt is strategic planning - which is traditionally viewed as setting a long-term direction based on sound predictions, analysis of options, and key decisions about the future of an organisation. Regrettably, water utilities, traditionally dominated by the engineering profession, often lack the necessary tools and capabilities to carry out strategic planning. The training of most water utility managers, although thorough in the functional areas of engineering, accounting or human resources, is often insufficient in strategic concepts, frameworks and tools. In addition, most utilities, while embracing strategic planning concepts, have attempted to embark on strategies 
without sufficient institutional analysis, internal participation, and adequate commitment of resources.

With the growing urban populations, water utilities in developing countries must adapt quickly to reduce the growing service gap, by reducing unaccounted for water, increasing revenues to cover operation costs, and expanding services to the urban poor. In addition, utilities must also adapt to the changing institutional and policy environment in which they operate. Given these uncertainties, this paper offers a strategic planning methodology to assist utilities in developing plans and articulating strategic actions to improve their performance and survival in an ever-changing environment. The framework for the methodology is explained and case studies are presented to illustrate how it can be applied to develop utility performance improvement plans. The research that led to the framework arose from a capacity building partnership project between the Water Utility Partnership of Africa (WUP), Severn Trent water (a UK water utility), Loughborough University (UK) and six African water utilities in Kenya, Uganda, Tanzania, Congo, Benin, and Lesotho. The methodology represents a new way for public water utilities to transform themselves and improve performance by addressing the internal and external problems they face.

\section{Management Challenges Facing Water Utilities}

Water utilities in developing countries differ greatly in terms of size, organisational culture and operating environments. However, a number of shared problems can be identified. First, the inefficiencies of water utilities are a major cause of poor access to water services in developing countries. In many systems, as much as a third of 
production is lost (through physical and commercial losses), and revenues are insufficient to cover operating costs let alone expand service coverage (World Bank, 2004). Indeed, for African utilities, reduction of unaccounted for water remains one of the major challenges. These problems are compounded by the general lack of a commercial orientation in utility management, and inappropriate tariffs regimes. With the growing urban population reported in many developing countries, water utilities need to quickly adopt robust plans and strategic actions to improve operational efficiency and reduce the service gap. Secondly, many utilities in developing countries lack effective management information systems to allow adequate monitoring and evaluation. Many of them rarely collect data systematically to assess their own performance in order to design operational improvements (Water Utility Partnership, 2000). As a result, both those responsible for service delivery, and those willing to support them lack the necessary information to design measures and investments to improve service delivery.

A third challenge facing water utilities in developing countries relates to generic utility management issues. Most utilities, despite having the legal mandate to supply water to all urban inhabitants, lack clearly articulated vision or mission statements, sound management structures, and human resource capacity to enable them fulfill their mandate. Given these challenges, governments supported by their development partners have sought to implement policy, regulatory and institutional reforms to create incentives for utilities to be more efficient, accountable, commercially oriented, and customer-focused. Such reforms would undoubtedly require utilities to change both their structures and strategy. Utility managers can draw on strategic 
management concepts to ensure that such changes are relevant, cost effective and transforming.

\section{Strategic Management Concepts}

Strategic management is 'concerned with determining the future direction of an organisation and implementing decisions aimed at achieving the organisation's long and short-term objectives' (Boseman and Phatak, 1989, p.4). Hence, the entire strategic management process can be said to comprise two major dimensions that are interlinked: strategic planning and strategy implementation and control. In strategic planning, strategic decisions are made concerning the organisation's mission and vision, its objectives and targets, as well as methods for achieving the objectives and targets. Strategic planning and corporate planning are sometimes used interchangeably in the literature to refer to an extensive organisation-wide resource allocation process (Jennings, 1999). On the other hand, strategy implementation and control is about translating strategic decisions into concrete actions necessary to achieve desired levels of performance.

Figure 1 shows a basic strategy model illustrating the context and consequence of strategic decisions. It shows that in making these decisions, managers attempt to find an optimal match between the internal resources and capabilities available within the utility and the external environmental threats and opportunities, in order to come up with a strategic plan, whose efficacy translates into some level of performance. Successful implementation of the strategic plan requires effective tactical or operational or planning. - i.e. making a variety of managerial and operational 
decisions (such as the type of organisation structure, leadership styles, management information systems, monitoring and evaluation systems) used to ensure that the utility’s objectives are achieved efficiently.

\section{Insert Figure 1 here}

Figure 1 also shows the importance of distinguishing strategic from tactical considerations in planning. Whereas a strategic plan maps out the future direction of the water utility, covering a period beyond the next fiscal year (usually three to five years), the tactical plan (usually covering a period of one year or less) ensures that the utility operates in the most efficient way, maximising its chances of success in delivering the strategic plan.

Although water utilities in developing countries have a legal mandate to supply water to all people living in their cities, they have not performed to the required standards, largely because their strategic plans are not complemented by effective tactical plans. Even with a clear corporate strategy, a utility without an effective tactical plan would only survive - serving only a small percentage of people, marginally breaking even, and not being able to invest in expanding service coverage or improving service quality. Managers need to develop a longer-term view of the organisation, while at the same time attending to the day-to-day management aspects. A utility with a clear corporate strategy and an effective tactical plan would thrive - serving more people and generating enough revenue for expansion. Water utility managers would benefit from a methodology that aligns the process of corporate strategic planning with 
identified performance problems, incorporating an in-depth operational plan to achieve performance improvements.

\section{Strategic Planning Framework for Water Utilities}

The foregoing strategic management concepts can be used to derive a solutionoriented planning framework for water utilities. Figure 2 shows the planning framework derived from the basic strategy model (Bourgeois, 1996). In its simplest form, the purpose of a solution-oriented strategic plan is to answer four central questions, i.e.

(i) Where is the utility now?

(ii) Where does the utility want to be?

(iii) How might the utility get there?

(iv) How does it ensure success?

\section{Insert Figure 2 here}

The first question is concerned with providing a complete situational analysis of the utility, including both performance and institutional aspects. A performance audit is a comprehensive and objective audit and review of the current state of utility performance and practice, measured against key performance indicators. Institutional analysis is concerned with answering questions relating to the utility’s external and internal environment, such as: what are the laws and controls (i.e. regulatory frameworks and control mechanisms), what are the incentives, what are the 
stakeholders and their roles, responsibilities and relationships; and what is the management culture (Grigg, 2005). Both performance audit and institutional analysis provide a helpful way to perform a gap analysis (i.e. a comparison of an existing situation with a desired one), which aids the next stage in the planning process.

The second question in the framework is concerned with setting utility objectives and targets. Based on the information collected in the situation analysis, performance objectives and targets for the utility can be developed, clarified and aligned with the utility’s mission and vision. For instance, a progressive urban water utility typically has the objective to improve service provision to all groups of existing and potential customers, while meeting its financial objectives. An additional strategic objective for a progressive water utility should be to meet the social good in terms of providing water to those currently not served by the utility (Njiru and Sansom, 2003). Whatever objectives are agreed, it is important that corresponding targets are specific, measurable, achievable, realistic and time bound.

The last two questions in the strategic planning framework are about operational planning, i.e. what concrete actions will the utility take to achieve its performance objectives and targets, and how will they be monitored and evaluated. Similar to targets, proposed actions should be specific, measurable, achievable, realistic and time-bound, in order to allow for effective monitoring and evaluation. The action plans should typically cover all aspects of utility management, such as: (i) review of existing roles, responsibilities and organisational structure; (ii) human resource management; (iii) management information systems; (iv) customer services 
management; (v) operation and maintenance management; (vi) reduction of unaccounted for water; (vii) capital investment; and (viii) financial management

Recent application of the framework in six urban water utilities in Africa has shown that it provides a systematic and repeatable standalone methodology that can be used by different utilities to develop comprehensive strategic work plans (Kayaga et al., 2006; Mihayo and Njiru, 2006; Sekhonyana et al., 2006; Tumuheirwe et al., 2006). The strategic work plan would address a variety of utility management issues, with the aim of improving performance and enabling the utility to achieve its short, medium and long-term objectives. In the following sections, we illustrate the practical application of the methodology using case studies from the African water utilities.

\section{Developing Utility Performance Improvement Plans: Case Studies}

\subsection{Case Background}

The action research that led to the strategic planning framework described in the previous section arose from a capacity building partnership project between the Water Utility Partnership of Africa, Severn Trent water (a UK water utility), Loughborough University (UK) and six African water utilities in Kenya, Uganda, Tanzania, Congo, Benin, and Lesotho. The Water Utility Partnership (WUP) is an organisation established to help water utilities in Africa to improve their performance and achieve economic and environmentally sustainable service delivery. WUP works by building partnerships among African water supply and sanitation utilities and other key sector institutions, to create opportunities for sharing experiences and capacity building. In 
order to achieve its objectives, WUP, supported by the Swedish International Development Agency (SIDA), initiated a project in 2001 aimed at improving utility management and reduction of unaccounted for water. This project formed part of WUP’s Action Programme designed to meet its objectives of improving utility performance, improving services to the urban poor and creating a framework for collaboration among water utilities and various training and research organisations.

The partnership arrangement was aimed at helping African water utilities to increase their performance by improving their operations and management. The project provided management skills training and technical support to six water utilities in six African countries. The participating utilities included: National Water and Sewerage Corporation (NWSC) in Entebbe (Uganda); Kisumu Water and Sewerage Company (KIWASCO) in Kisumu (Kenya), Mwanza Urban Water and Sewerage Authority (MWAUWASA) in Mwanza (Tanzania), Water and Sewerage Authority (WASA) in Maseru (Lesotho), Société Nationale des Eaux du Benin (SONEB), in Cotonou (Benin) and Société Nationale de Distribution d’Eau (SNDE) in Brazzaville (Congo). Severn Trent Water and Loughborough University’s Water Engineering and Development Centre (WEDC) constituted the action research team.

The process to select participating utilities started in the first half of 2000. Water Utility Partnership (WUP) sent information to all its member water utilities explaining the purpose and scope of the project, and invited interested utilities to show willingness to participate. One of the preconditions for participation was spelt out as the willingness and capacity of the utility to provide minimal contributions to the project costs, in terms of staff time and travel expenses. Over twenty utilities initially 
showed interest in the project. WUP used the following main criteria to narrow down the number to six:

a. Preference for utilities with relatively high proportion of unaccounted-for-water (UfW), where the project would easily demonstrate impact,

b. Preference for relatively small utilities serving not more that 500,000 people, where impact would more easily be achieved using the limited financial resources, in the given time frame, and

c. Achievement of a right balance between English-speaking and French-speaking countries.

The capacity building project was undertaken in two phases. Phase one commenced at the end of 2000, and involved selection and performance audit of representative African utilities. Phase two of the project commenced in July 2003, and mainly involved developing critical management skills to enable participating utilities prepare their performance improvement plans and action plans for the reduction of unaccounted for water. A capacity needs assessment carried out in the course of this action research identified deficient areas and carried out training in (i) institutional analysis and change management; (ii) customer relations management; (iii) financial management; (iv) human resource management; (v) operation and maintenance management; and (v) management of UfW.

It was during this second phase that the strategic planning framework and methodology was developed and tested. Participating utilities used the framework 
and methodology to prepare performance improvement plans (PIP). A performance improvement plan is simply a road map to achieve stated utility objectives, and should be able to: (i) define the scope of the utility's activities in terms of what it will do, and what it will not do; (ii) match the utility's activities to the environment in which it operates, so that it optimises opportunities and minimises threats; and (iii) match the utility's activities to its resources capacity such as staff, finance, infrastructure, and technology.

In the following sections, we draw on the experiences of the six participating water utilities to point out some of the key considerations and potential benefits of using the strategic planning framework to prepare a utility PIP. We begin by discussing the question of who should be involved in the process of PIP development. Then, a discussion of key considerations in answering the four central questions in the planning framework is given, together with a generic structure of the PIP document. We conclude the section by highlighting some of the benefits a water utility might derive from engaging in a PIP process, the constraints to applying the framework, and the key lessons learnt from the case studies. Due to space limitations, the case examples used to illustrate the different aspects are drawn from only four participating utilities in Uganda, Kenya, Tanzania and Lesotho.

\subsection{Who should be involved in PIP Development?}

The process of developing a PIP should be participatory, involving utility staff of all levels of seniority, gender, discipline and age. This leads to a shared focus, and enhances staff commitment during implementation (Tumuheirwe et al., 2006). 
However, it is important that a core team of senior managers from different disciplines within the utility is formed and charged with responsibility for the PIP preparation. Within this core team, a focal person should be appointed whose role is to lead communication, organization and delivery of the PIP development. For instance, in the case of Kisumu, the lead role was assigned to a 'PIP Champion'- who was given responsibility for moving the process forward and for data collection, analysis, preparation of reports and coordination of regular meetings with key staff to review progress and identify future actions.

\subsection{Where is the utility now?}

The first step in the planning process is to provide a complete situational analysis of the utility, including both performance and institutional aspects. The necessary information can be obtained using relevant appraisal techniques such as StrengthWeakness-Opportunities-Threats (SWOT) and Political-Economic-SocialTechnological (PEST) analyses, as they assist in understanding and summarizing the institution's environment and performance gaps. A SWOT analysis identifies the organizational strengths, weaknesses, opportunities and threats, and enables the organisation to determine the set of key success factors - i.e. those activities or areas in which it must be especially proficient to succeed by fully exploiting the opportunities available to it and combating the threats that endanger it (Boseman and Phatak, 1989). An example of a SWOT analysis carried out by the senior and middle staff of Maseru Water and Sewerage Authority (WASA) -Lesotho is given in Table 1. 
A utility attempting to analyse its own SWOT must also look inward and evaluate its own capabilities in terms of skills and competences present that would enable it to do especially well in those areas identified as key success factors. Indeed, the objective matching of an organisation's capabilities and resources against key success factors enables a good estimate of its strengths and weaknesses. On the other hand, a PEST analysis considers political, economic, social and technological issues that could affect utility performance in the long term. Such an analysis ensures that resulting plans are aligned with any changing circumstances relating to political, economic, social and technological issues. It helps to avoid unrealistic or untenable strategies due to external circumstances, thus allowing strategic plans to adapt to external realities.

\subsection{Where does the utility want to be?}

Based on the information collected in the situation analysis, performance objectives for the utility can be developed and clarified in order to address identified weaknesses, reinforce strengths, seize opportunities, and mitigate against effects of perceived threats. The key consideration here is that objectives must have corresponding targets, and the targets should be specific, measurable, achievable, realistic and time bound. Box 1 shows an example of performance targets highlighted by National Water and Sewerage Corporation in Entebbe (Uganda) during the development of the utility’s five year (2004-2009) performance improvement plan. In setting performance objectives and targets, utility managers need to ensure that objectives and targets are in line with the mission and vision of the utility. 


\subsection{How might the utility get there?}

Following the process of setting objectives and targets, the next important stage in performance improvement planning is to identify concrete actions that the utility will take to achieve its performance objectives and targets. This involves making a variety of managerial and operational decisions relating to a broad range utility management issues as given in Figure 2. One of the main focus points for the participating utilities was to achieve a reduction in the percentage of unaccounted for water (UfW). Thus the PIPs prepared by the different utilities incorporated an action plan for reduction of UfW. Box 2 shows an example of concrete actions identified by the Water and Sewerage Authority in Maseru (Lesotho) for the reduction of unaccounted for water. It can be noted that utility action plans for UfW reduction did not focus purely on reducing leakage but also considered other strategies such as: (i) improving billing accuracy and efficiency; (ii) ensuring available of appropriate materials; and (iii) capacity building.

\section{Insert Box 2 here}

\subsection{How does the utility ensure success?}

In order to ensure the success of a PIP, it is important to provide continuous feedback on the utility's progress towards performance improvements at all levels through an effective monitoring and evaluation mechanism. This enables management to identify actual and potential success and failure early enough to facilitate timely 
adjustments. An example of a monitoring framework is provided by National Water and Sewerage Corporation in Entebbe-Uganda (Figure 3).

\section{Insert Figure 3 here}

In this arrangement, all activities are initiated and implemented by a team from the section in charge (or a cross function team composed of members with the right skills from different sections). The PIP task force is made up of heads of sections responsible for coordinating activities and evaluating performance in line with the agreed targets, and reporting to the Area Manager on a monthly basis. The Area Manager oversees the implementation of the PIP as the accounting officer and prepares quarterly and annual reports as appropriate. The Research, Monitoring and Evaluation Department at the Head Office provide strategic guidance on the effective implementation of the PIP.

\subsection{Generic PIP Structure}

It is difficult to prescribe how a PIP should be structured, as the contents depend on the context in which it is developed. However, following a review of the PIPs developed by the six utilities that participated in the project, a generic structure was identified. An abridged version of such a structure is given in Box 3. In the section on conclusion, the participants and/or authors may document what challenges they faced, what lessons were learnt during the preparation of the PIP, and what aspects of the process needed improvement. Details of the supporting documents could be placed in 
Appendices. Examples of such documents are detailed work plans for each of the performance improvement strategies. It would also be advisable to write an executive summary of the PIP report, for the benefit of top managers and other internal and external publics who, for various reasons may not be able to read all the details of the document.

Insert Box 3 here

\subsection{Benefits of Utility Performance Improvement Planning}

A water utility can derive many benefits from a performance improvement plan. First, it creates a platform for a common focus, leading to a clear understanding of the utility's vision, mission and objectives. Secondly, the planning process itself helps to bring out structural deficiencies in the utility, which could trigger enabling legislation. Thirdly, the process it self enhances accountability and transparency, thus creating confidence among internal and external stakeholders. Finally, and most importantly, a PIP is a tool to implement significant process improvements and changes to enable the utility achieve higher levels of performance. For instance, all the six utilities that participated in the WUP capacity building project now have a better vision of what needs to be done to improve their overall performance, and as a consequence enable a better level of service to be provided to their customers.

Although, full implementation of the plans is dependent upon adequate financial resources being made available (either from within the utility or externally sourced), participating utilities have registered some achievements as a result of the PIP process 
in general. Initial results emerging from the participating utilities include: (i) organisational restructuring from traditional civil service style to more commercially structures; (ii) review of management information systems in light of objectives and targets set; (iii) enhanced understanding of the importance of UfW reduction; (iv) better planning enhanced by financial modelling; (v) significant reductions in unaccounted for water for some utilities (e.g. Mwanza and Kisumu); and (vi) increased capacity of senior and middle management staff in strategic planning.

It was difficult to obtain baseline performance indicators from most of the participating utilities at the start of the project, to enable us evaluate the objectively quantifiable performance trends. One exception is Mwanza Urban Water and Sewerage Authority, which already had a robust management information system by the time the project started. Table 2 shows the basic performance indicators at the start of the project in 2002/03 and three years later in 2005/06. The table shows a positive performance trend in all the cited indicators, which may partly be attributed to the strategic planning process (Mihayo and Njiru, 2006).

Insert Table 2 here

\subsection{Constraints and Key Lessons}

Although a water utility can derive many benefits from strategic planning, applying the strategic planning methodology described in this paper is not without challenges. First, successful implementation of the plans requires adequate financial resources. For most cash strapped utilities in developing countries, this is a major challenge to adapting the methodology. However, the PIP itself has the potential to create 
confidence among external funding organisations, thus improving the utility's creditworthiness.

Second, for water utilities that have traditionally been run on a civil service/administrative style of management, introducing strategic planning requires a major culture change within the organisation. Spearheading such change requires committed senior and middle managers equipped with the necessary management skills. For some of the case study utilities, the PIP process was not adequately mainstreamed within the organisation and in some instances top management commitment was low.

Third, setting a long-term strategic direction for an organisation often requires good quality baseline information. Such information was often lacking in the case study utilities, as many of them rarely collected data systematically to assess their own performance. It was therefore difficult in some instances for utility managers to make sound predictions, analyse options, and key decisions about the future.

In spite of the constraints, a key lesson emerging from the case studies is that comprehensive strategic planning is a crucial part of efforts to improve utility performance. The framework discussed in this paper can be adapted by other utilities to develop comprehensive strategic plans addressing a variety of technical and management issues and enabling the utility to achieve its short, medium and longterm objectives. However, for most water utilities in developing countries, additional resources are needed for capacity building in order to develop the necessary critical management skills. 


\section{Conclusion}

This paper has attempted to provide a strategic planning framework and methodology for urban water utilities. The paper has focused on developing countries - where there are dismal levels of access to safe water and sanitation services, and water utilities have not performed as expected. Although the institutional set up of utilities in developing countries differs greatly, a number of shared management challenges can be identified, such as inefficiency in operations, high proportions of unaccounted for water, ineffective management information systems, and general lack of a commercial orientation. In addition, public water utilities are operating in a constantly changing policy and institutional environment, with governments committed to implementing reforms aimed at making utilities more efficient, accountable and commercially oriented. Such reforms would undoubtedly require utilities to change both their structures and strategy.

The authors have urged that public utility managers need to draw lessons from strategic management concepts practiced in the private sector in order to develop relevant, cost-effective and transforming strategic plans. A framework for strategic planning has been provided, which in its simplest form, is composed of four central questions: where are we now, where do we want to be, how might we get there and how do we ensure success? Providing comprehensive answers to these four basic questions, through internal participation, generates a relevant and cost-effective road map for the utility, which integrates both strategic and tactical planning. Case studies and examples have been given to illustrate how the framework can used in practice. While strategic planning concepts are not entirely new, the contribution of this 
framework is to offer a systematic and repeatable methodology for utilities seeking to develop meaningful and useful performance improvement plans.

\section{Acknowledgements}

This action research was sponsored by the Swedish International Development Cooperation Agency (SIDA) as part of a study for the benefit of developing countries. The views expressed are not necessarily those of SIDA. The authors are grateful for the efforts of all members of the international research team, particularly those from Severn-Trent Water; the Water Utility Partnership of Africa (WUP-Africa); and management and staff of the participating utilities, for their cooperation and their dedication during the research study. 


\section{References}

Boseman, G. and Phatak, A. (1989). Strategic Management: Texts and Cases. John Wiley, New York

Bourgeois, L. J. (1996). Strategic Management: From Concepts to Implementation. The Dryden Press, Fort Worth, TX,

Budds, J. and McGranahan, G. (2003). "Are the Debates on Water Privatisation Missing the Point? Experiences from Africa, Asia and Latin America." Environment and Urbanization 15(2): 87-113.

Grigg, N. S. (2005). "Institutional Analysis of Infrastructure Problems: Case Study of Water Quality in Distribution Systems." Journal of Management in Engineering 21(4): 152-158.

Jennings, D. (1999). “Corporate Planning: Post-Privatisation Change in a UK Electricity Utility” Utilities Policy, 8(4), 223-232

Kayaga, S., Njiru, C., Itiko, M. and Onyango-Awin, J. (2006). Improving Utility Management: Case Study from Kisumu, Kenya, in: Kayaga, S. (Ed.), Proceedings of the 31st WEDC International Conference, WEDC, Loughborough University, UK, pp.172-175. Available at: http://wedc.lboro.ac.uk/conferences/pdfs/31/Kayaga.pdf [accessed 21 June 2006]

Lam, P. and Chan, Y. (1998). "Privatising Water Supply in Hong Kong”. Utilities Policy, 7(2), 95-105 
Mihayo, Z. and Njiru, C. (2006). Improving Utility Management: Case Study of MWAUWASA, Mwanza Tanzania, in: Kayaga, S. (Ed.), Proceedings of the 31st WEDC International Conference, WEDC, Loughborough University, UK, pp. 196199. Available at: http://wedc.lboro.ac.uk/conferences/pdfs/31/Mihayo.pdf [accessed 21 June 2006]

Njiru, C. and Sansom, K. (2003). "Strategic marketing of water services in developing countries." Proceedings of the Institution of Civil Engineers. Municipal Engineer 156(2): 143-148.

Sekhonyana, S., Pholo, M. T. and Fisher, J. (2006). Improving Utility Management: Case Study from Lesotho, in: Kayaga, S. (Ed.), Proceedings of the 31st WEDC International Conference, WEDC, Loughborough University, UK, pp.254-257. Available at: http://wedc.lboro.ac.uk/conferences/pdfs/31/Sekhonyana.pdf [accessed 21 June 2006]

Tumuheirwe, S., Lutaaya, M. and Kayaga, S. (2006). Improving Utility Management through Partnership and Capacity Building, in: Kayaga, S. (Ed.), Proceedings of the 31st WEDC International Conference, WEDC, Loughborough University, UK, pp.266-269. At: http://wedc.lboro.ac.uk/conferences/pdfs/31/Tumuheirwe.pdf [accessed 21 June 2006]

Water Utility Partnership (2000). Performance Indictors of Some African Water Supply and Sanitation Utilities. Water Utility Partnership. Abidjan, Cote-d'Ivoire Wilson, R.M.S. and Gilligan, C., (1997). Strategic Marketing Management, $2^{\text {nd }}$ edition, Butterworth-Heinemann, London 
World Bank (2003). Private Participation in Infrastructure: Trends in Developing Countries in 1990-2001. The World Bank, Washington DC

World Bank (2004). Reforming Infrastructure: Privatisation, Regulation and Competition. World Bank Policy Research Report. Oxford University Press, Oxford

Zuleta, F., Merlano, A., Alvarez, A., Montoya, M. and Restrepo, E. (2005).

"Modernisation of the water utility company of Bogota (Colombia): An example of public and private participation for solving management problems of water companies in developing countries." Journal of Water and Science and Technology: Water Supply 5(2): 107-113. 


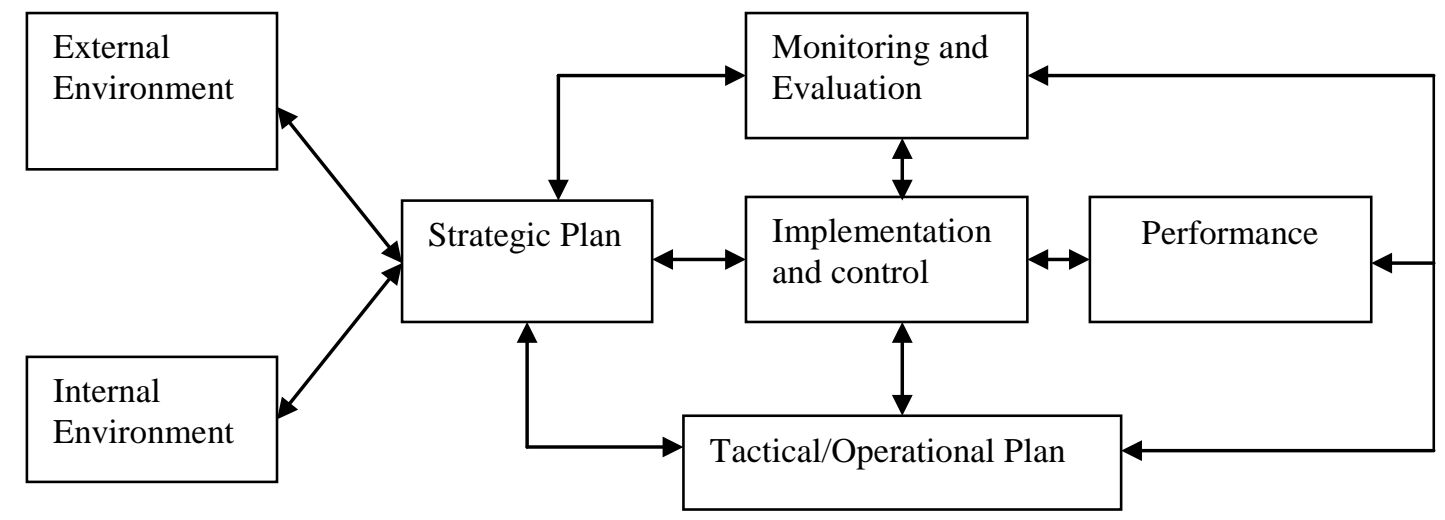

Fig. 1: The basic strategy model (adapted from Bourgeois, 1996) 


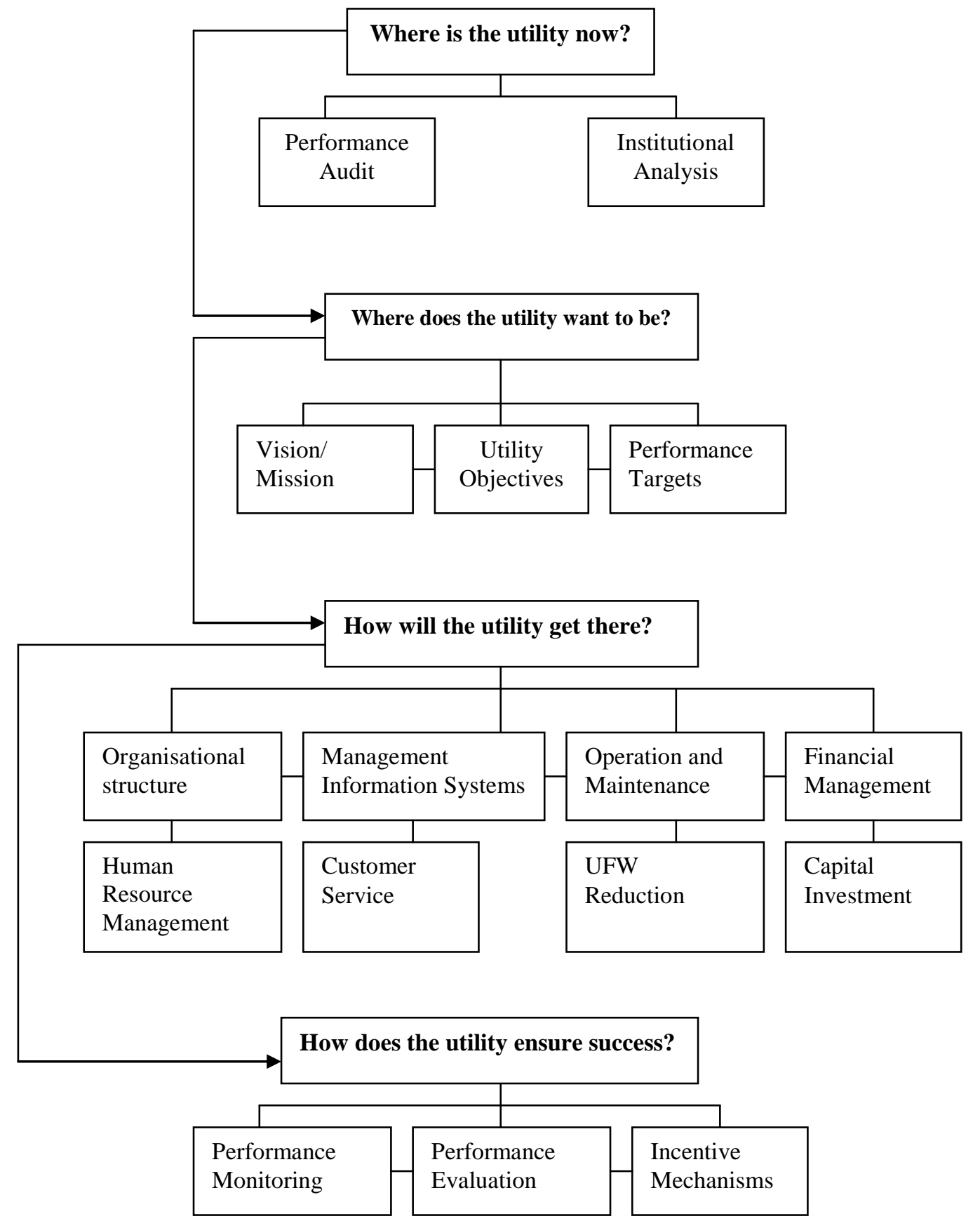

Fig 2: Strategic planning framework for water utilities (Adapted from Wilson \& Gilligan, 1997) 


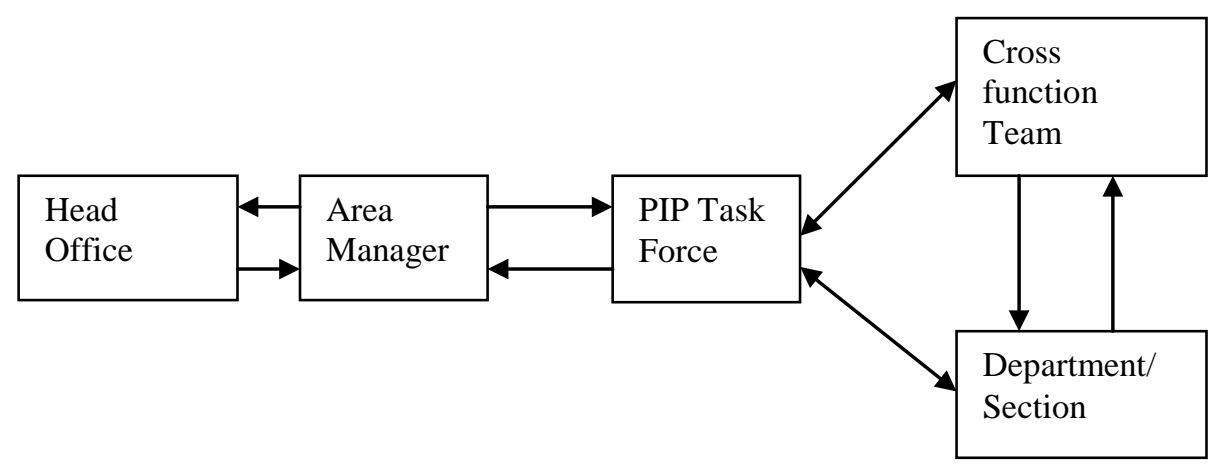

Fig 3: Conceptual monitoring framework for NWSC, Entebbe, Uganda 
Table 1: Abridged results of SWOT analysis by WASA, Maseru Lesotho (October 2003)

\section{Strengths}

- Business monopoly

- Large customer base

- Staff with good sector knowledge

- Available resources

- Well-paying customers

- Financial stability

\section{Weaknesses}

Inadequate leadership

- Weak corporate governance

Poor communication

Poor customer service

Low-level of revenue

Corruption and fraud

High level of unaccounted for water

Age and level of infrastructure maintenance

Centralised decision making

Inaccurate meter readings

Inadequate management information systems

Inability to meet demand

\section{Opportunities}

- Monopoly status

- High water demand

- Demand for convenient payment methods

- Links with other institutions

- Donor assistance

- Benefits of proximity to South Africa

- Environmental Act

- Available good quality water

\section{Threats}

Droughts

Privatisation of utilities

- Crime

- Legal impediments

- Environmental pollution

Industrial disputes

High mortality rate

Unemployment

Old loans

Lack of union

Inadequate water sources 
Table 2: Key Performance Indicators for Mwanza Urban Water and Sewerage Organisation (MWAUWASA), Tanzania for the period 1996/97 to 2005/06 (Adapted from Mihayo \& Njiru, 2006)

\begin{tabular}{lcccc}
\hline Indicator & $\mathbf{1 9 9 6 / 9 7}$ & $\mathbf{2 0 0 2 / 0 3}$ & $\mathbf{2 0 0 3 / 0 4}$ & $\mathbf{2 0 0 5 / 0 6}$ \\
\hline Water produced/yr $\left(000 \mathbf{\prime}^{3}\right)$ & No data & 14,279 & 14,337 & 14,280 \\
Unaccounted for water & $76 \%$ & $57 \%$ & $50 \%$ & $40 \%$ \\
Water supply area coverage & No data & $70 \%$ & $72 \%$ & $82 \%$ \\
Customer base (no. of connections & 8,000 & 14,515 & 16,303 & 21,340 \\
Metered connections & $1 \%$ & $76 \%$ & $89 \%$ & $97 \%$ \\
Av. water supply hours/day & 12 & 20 & 22 & 21 \\
Sewerage service area coverage & No data & $7 \%$ & $7 \%$ & 8.5 \\
Staff per 1000 connections & 20 & 14 & 12 & 10 \\
Operating ratio & No data & 0.85 & 0.96 & 0.8 \\
Days receivable ration & $>300$ & 206 & 180 & 120 \\
Revenue collection efficiency & No data & $94 \%$ & $95 \%$ & $97 \%$ \\
\hline
\end{tabular}




\section{Box 1: Performance Targets for National Water and Sewerage Corporation, Entebbe - Uganda (2005)}

1. Apply best practice management techniques that ensure overall reduction of Non-revenue water from $30-20 \%$ by 2009

2. Institute measures that will significantly increase annual revenue to USHS 5billion from the current Ushs 1.9 billion and ensure 100\% compliance with WHO standards

3. Ensure value for money capital investments with a payback period of not more than 15 years

4. Carry out effective process control to ensure quality at all stages of water production, water supply and sewage treatment and ensure 100\% compliance with WHO standards

5. Ensure viability of NWSC Entebbe through maximisation of revenues and optimization of costs to no more than $45 \%$ working ratio level

6. Develop and implement an integrated Management Information System that supports and addresses business needs of the Entebbe Area

7. Promote good customer service and ensure customer satisfaction level of at least $98 \%$

8. Put in place systems that will ensure increased services coverage especially to low income earners from $64-95 \%$.

Box 2: Elements of WASA (Lesotho)'s Action Plan for Reducing UfW from $37 \%$ to $25 \%$ by 2009

Install bulk meters at production points and service reservoirs

- Ensure that quarterly drop tests are conducted at service reservoirs

- Update network maps

- Install district meters

- Carry out active leak detection activities

- Carry out pressure zoning and install pressure reducing valves

- Replace uneconomical-to-repair pipeline sections in a rational manner

- Digitise the water network and pressure maps

- Acquire network modelling software

$\circ \quad$ Carry out planned preventative maintenance of network

- Ensure all connections are metered

- Improve the accuracy of meters and meter readings

- Improve accuracy of bills

- Carry out surprise visits to sampled disconnected users to discourage illegal use 


\section{Box 3: Generic PIP Structure}

A typical PIP comprises the following sections:

1. Introduction: a short brief about the utility in question, covering a summary of its development and reasons why the PIP is being prepared

2. Statement of utility mandate, vision and vision

3. Situation analysis (where is the utility now?): a comprehensive audit using indicators to describe its current status, including, for example, results of SWOT and PEST

4. PIP objectives and timeframe (where does the utility want to be?): agreed performance indicators, standards and targets should be stated, along with any assumptions

5. Performance improvement strategies (how might the utility get there?): Several important sub-headings may fall under this: i.e. roles, functions and responsibilities; human resources management strategy; management information systems, customer services management strategy; operations and maintenance strategy; capital investment strategy; financial management strategy; reduction of UfW; revenue and collections; water quality improvement strategy; effluent quality improvement

6. Performance monitoring and evaluation (how does the utility ensure success?): this section described monitoring mechanisms, performance review and evaluation criteria, and incentives mechanisms.

7. Conclusions

8. Appendices 